\title{
Utilização de Cinco Espécies de Eucalyptus para a Produção de Painéis OSB
}

\author{
Thiago Souza da Rosa ${ }^{1}$, Rosilani Trianoski ${ }^{2}$, Setsuo Iwakiri², \\ Ghislaine Miranda Bonduelle ${ }^{2}$, Huan Pablo de Souza ${ }^{3}$

\footnotetext{
${ }^{1}$ Programa de Pós-graduação em Engenharia Florestal, Universidade Federal do Paraná - UFPR, Curitiba/PR, Brasil ${ }^{2}$ Departamento de Engenharia e Tecnologia Florestal, Universidade Federal do Paraná - UFPR, Curitiba/PR, Brasil ${ }^{3}$ Equipe de Tecnologia Florestal, Stora Enso Florestal RS Ltda, Rosário do Sul/RS, Brasil
}

\begin{abstract}
RESUMO
O objetivo deste estudo foi avaliar a viabilidade técnica da utilização de cinco espécies de Eucalyptus, provenientes de plantios com ciclo de curta rotação, para produção de painéis OSB. As espécies estudadas foram: Eucalyptus benthamii, Eucalyptus dunni, Eucalyptus grandis, Eucalyptus saligna, Eucalyptus urograndis, a mistura entre elas e o Pinus taeda como testemunha. Os painéis de Eucalyptus apresentaram maior estabilidade dimensional quando comparados com os painéis testemunha. Os painéis compostos pelo mix de espécies apresentaram estabilidade dimensional semelhante aos demais produzidos com as espécies de Eucalyptus puras. No ensaio de flexão estática os valores de MOR não apresentaram diferenças estatísticas entre os tratamentos, porém, para MOE, os painéis de Eucalyptus apresentaram valores estatisticamente superiores quando comparados aos da testemunha. Na tração perpendicular os painéis produzidos com Pinus taeda apresentaram valor médio superior às demais espécies. Todas as espécies assim como a mistura entre elas mostraram viabilidade técnica para produção de painéis OSB.
\end{abstract}

Palavras-chave: curta rotação, painéis de partículas orientadas, espécies de rápido crescimento, painéis estruturais e não estruturais, fenol-formaldeído.

\section{Use of Five Eucalyptus Species to the Manufacture of Oriented Strand Board}

\begin{abstract}
This study aimed to assess the technical feasibility of using five Eucalyptus species to manufacture Oriented Strand Boards. The species studied were: Eucalyptus benthamii, Eucalyptus dunni, Eucalyptus grandis, Eucalyptus saligna, Eucalyptus urograndis the mixture between them and the Pinus taeda as control. The panels composed by a mix of species showed dimensional stability similar to others produced with Eucalyptus pure species. The panels of Eucalyptus had lower water absorption and thickness swelling values to the controls panels. In static bending, the MOR values did not show statistics differences between treatments, however, for MOE the Eucalyptus panels showed statistically higher values when compared to control specie. In internal bond, the panels produced with Pinus taeda showed higher average value than the other species. All species as well as the mixture showed technical feasibility for the production of Oriented Strand Board.
\end{abstract}

Keywords: short rotation, oriented strandboard, fast growing species, structural and non-structural panels, phenol-formaldehyde. 


\section{INTRODUÇÃO}

Segundo o Sistema Nacional de Informações Florestais (SNIF, 2013), há pouco mais de um século de introdução no país, o gênero Eucalyptus possui a maior área com floresta plantada no Brasil, atingindo cerca de 5.473.176 hectares em 2013. Esse número foi alcançado pelo fato de o país apresentar atualmente tecnologias avançadas na silvicultura do Eucalyptus podendo atingir rendimento de cerca de $60 \mathrm{~m}^{3} / \mathrm{ha} \mathrm{em}$ rotações de 7 anos.

A principal matéria-prima utilizada no setor de painéis reconstituídos é a madeira de Pinus, principalmente o Pinus taeda, proveniente de florestas plantadas (Mendes, 2010), no entanto outras espécies vêm sendo pesquisadas para se tornarem alternativas na produção de OSB (Gorski, 2014) e passarem consequentemente a integrar e aumentar sua participação na gama de matéria-prima disponível para o segmento de painéis.

A utilização de madeiras do gênero Eucalyptus ainda é pequena nesse setor. Segundo a ABRAF (2013), do total de florestas plantadas apenas 4,3\% são direcionados para esse segmento. A maior parte da madeira dessas florestas é utilizada principalmente nos setores de energia e celulose e papel, por terem alta demanda de espécies de fibra curta para a geração dos seus produtos.

De acordo com Iwakiri et al. (2001), o aumento da oferta de matéria-prima com espécies com características como baixa massa específica da madeira e rápido crescimento é necessário, tendo em vista que também são requeridos grandes volumes de madeira no processo industrial de produção de painéis. As espécies do gênero Eucalyptus apresentam essas características, podendo ser uma alternativa viável para amenizar impasses resultantes da grande demanda de matéria-prima das indústrias de painéis reconstituídos. Além disso, diversos autores, como Iwakiri et al. (2008), Mendes et al. (2014) e Gorski et al. (2015), indicaram a partir de suas pesquisas que painéis OSB produzidos com espécies do gênero Eucalyptus apresentaram propriedades superiores aos painéis tradicionalmente produzidos com Pinus.

Dentre os principais tipos de painéis reconstituídos destacam-se os painéis de partículas orientadas (Oriented Strand Board - OSB). Desenvolvido nos Estados Unidos em torno de 1935, a produção experimental desse painel começou em 1963, expandindo-se posteriormente para a Europa em 1978 (Irle \& Barbu, 2010). No Brasil, mais precisamente em Ponta Grossa, PR, esse tipo de painel começou a ser produzido comercialmente em 2002, pela Masisa, indústria com capacidade produtiva de $350 \mathrm{mil} \mathrm{m}^{3} /$ ano, a qual, a partir de 2008 , passou a ser operada pela Lousiana Pacific (LP), maior fabricante de OSB no mundo (Vidal \& Hora, 2014).

Considerando-se a importância do setor de painéis reconstituídos, especialmente o OSB, para a economia nacional, a necessidade de ampliar a variedade de madeira para essa finalidade e a possibilidade de obtenção de painéis com propriedades superiores, este trabalho teve como objetivo avaliar a viabilidade da utilização de cinco espécies de Eucalyptus, puras e em mistura, para a produção de painéis estruturais OSB.

\section{MATERIAL E MÉTODOS}

\subsection{Matéria-prima}

Foram utilizadas, neste estudo, madeiras de Eucalyptus benthamii Maiden e Cambage, Eucalyptus dunni Maiden, Eucalyptus grandis Hill ex Maiden, Eucalyptus saligna Smith e Eucalyptus urograndis (Eucalyptus urophylla $x$ Eucalyptus grandis) provenientes de plantios de curta rotação com 7 anos de idade, localizados em Rosário do Sul, RS. Foram amostradas 5 árvores por espécie, as quais foram seccionadas em toras, desdobradas em blocos e posteriormente processadas para geração das partículas. Como testemunha foi utilizada a madeira de Pinus taeda L., procedente da região de Santa Catarina, com massa específica básica de $0,36 \mathrm{~g} / \mathrm{cm}^{3}$ e 21 anos de idade.

As partículas foram geradas com dimensões nominais de $80 \mathrm{~mm}$ de comprimento, $25 \mathrm{~mm}$ de largura e 0,6 $\mathrm{mm}$ de espessura, em picador de disco rotativo com 4 facas. As partículas foram secas em estufa a uma temperatura de $80^{\circ} \mathrm{C}$ até obterem um teor de umidade de aproximadamente $3 \%$ e, então, classificadas em peneira manual com aberturas de 9,52 mm para retirada de "finos".

Para a colagem dos painéis foi utilizada resina fenol-formaldeído, com teor de sólidos de 51,57\% e viscosidade de $503 \mathrm{cP}$, na proporção de $6 \%$ de sólidos base peso seco das partículas. Foi utilizado 
também $1 \%$ de emulsão de parafina a fim de reduzir a higroscopicidade dos painéis.

\subsection{Produção dos painéis}

Foram produzidos 21 painéis OSB, com dimensões de $48 \mathrm{~cm} \mathrm{x} 48 \mathrm{~cm} \times 1,25 \mathrm{~cm}$ e massa específica nominal de $0,70 \mathrm{~g} / \mathrm{cm}^{3}$, sendo 3 painéis para cada um dos 7 tratamentos. Os percentuais de camada externa e interna foram de 20:60:20.

Além da utilização das espécies na forma pura, foram produzidos painéis com a mistura das cinco espécies (mix) de Eucalyptus, numa proporção de $20 \%$ cada, e o tratamento testemunha (Pinus taeda). $\mathrm{O}$ delineamento experimental pode ser visualizado na Tabela 1.

As partículas foram levadas à encoladeira rotativa e submetidas à aplicação de resina fenol-formaldeído e emulsão de parafina por meio de um compressor a ar e pistola aspersora com recipiente dosador. Após a encolagem, o colchão de partículas foi formado orientando as camadas perpendicularmente por meio de uma caixa orientadora acoplada à caixa formadora com dimensões de $48 \mathrm{~cm} \mathrm{x} 48 \mathrm{~cm}$ x $20 \mathrm{~cm}$. Após a formação do colchão, foi realizada a pré-prensagem de forma manual para a remoção do ar e acomodação das partículas.

A prensagem dos painéis foi realizada em prensa hidráulica com $40 \mathrm{kgf} / \mathrm{cm}^{2}$ de pressão específica e a uma temperatura de $160^{\circ} \mathrm{C}$ por um período de 10 minutos.

Os painéis produzidos foram acondicionados em câmara climatizada com temperatura de $20^{\circ} \mathrm{C} \pm 3{ }^{\circ} \mathrm{C}$ e umidade relativa de $65 \% \pm 5 \%$ até atingirem o teor de umidade de equilíbrio. Após a estabilização dos

Tabela 1. Delineamento experimental.

Table 1. Experimental design.

\begin{tabular}{ccc|} 
Tratamento & Espécie & $\begin{array}{c}\text { Massa } \\
\text { específica } \\
\text { nominal } \\
\left(\mathbf{g} / \mathbf{c m}^{3}\right)\end{array}$ \\
\hline 1 & Eucalyptus benthamii & 0,70 \\
\hline 2 & Eucalyptus dunnii & 0,70 \\
\hline 3 & Eucalyptus grandis & 0,70 \\
\hline 4 & Eucalyptus saligna & 0,70 \\
\hline 5 & Eucalyptus urograndis & 0,70 \\
\hline 7 & Mix & 0,70 \\
\hline
\end{tabular}

*Mistura constituída de $20 \%$ de cada espécie de Eucalyptus. painéis, foram aparados $3 \mathrm{~cm}$ de cada borda, e então confeccionados os corpos de prova, os quais foram levados novamente à climatização para posterior realização dos ensaios físicos e mecânicos.

\subsection{Ensaios físicos e mecânicos}

Todos os ensaios físicos e mecânicos foram realizados seguindo os procedimentos das normas europeias: massa específica aparente (CEN, 2002d), absorção de água e inchamento em espessura (CEN, 2002b), flexão estática (CEN, 2002a) e tração perpendicular à superfície do painel (CEN, 2002c). A disposição de onde foram retirados os corpos de prova do painel pode ser visualizada na Figura 1.

Os resultados obtidos nos ensaios foram submetidos à análise de variância, e quando rejeitada a hipótese de nulidade, à comparação de médias de Tukey, todos ao nível de probabilidade de erro de 5\%. Os resultados foram, ainda, comparados com os requisitos sugeridos pela norma (CEN, 2006).

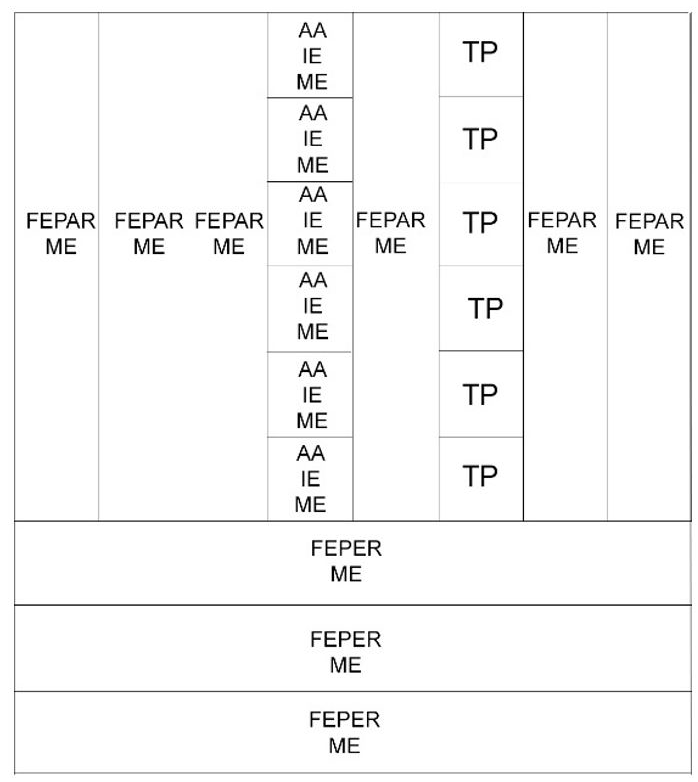

Figura 1. Disposição dos corpos de prova no painel. $\mathrm{ME}=$ massa específica aparente; $\mathrm{AA}=$ absorção d'água; $\mathrm{IE}=$ inchamento em espessura; $\mathrm{TP}=$ tração perpendicular; FEPAR = flexão estática paralela; FEPER = flexão estática perpendicular.

Figure 1. Disposition of samples on panel. $\mathrm{ME}=$ Specific gravity; $\mathrm{AA}=$ Water absorption; $\mathrm{IE}=$ Thickness swelling; TP = Internal bond; FEPAR = Parallel static bending; FEPER = Perpendicular static bending. 


\section{RESULTADOS E DISCUSSÃO}

\subsection{Propriedades físicas da madeira e dos painéis}

Os valores médios de massa específica da madeira, dos painéis e suas respectivas razões de compactação são apresentados na Tabela 2.

De acordo com os valores médios encontrados para a massa específica da madeira, as espécies podem ser classificadas, segundo Melo et al. (1990), como leves, por apresentarem valor de até $0,500 \mathrm{~g} / \mathrm{cm}^{3}$. Verifica-se ainda que, com exceção do E. grandis e da testemunha, as demais espécies apresentaram massa específica média ligeiramente superior ao valor sugerido por Cloutier (1998) para painéis OSB, que varia de $0,250 \mathrm{~g}$ a $0,450 \mathrm{~g} / \mathrm{cm}^{3}$.

Em relação à massa específica dos painéis, pode-se notar que não houve diferença estatística significativa entre os valores médios dos diferentes tratamentos. Isso indica que conseguiu-se obter uma boa homogeneidade para essa variável por meio do controle no processo produtivo, obtendo maior precisão na comparação entre as espécies.

Tabela 2. Valores médios de massa específica da madeira e do painel e razão de compactação.

Table 2. Average values of wood and panel density and compression ratio.

\begin{tabular}{lccc}
\multicolumn{1}{c}{ Espécie } & $\begin{array}{c}\text { ME madeira } \\
\left(\mathbf{g} / \mathbf{c m}^{3}\right)\end{array}$ & $\begin{array}{c}\text { ME painel } \\
\left(\mathbf{g} / \mathbf{c m}^{3}\right)\end{array}$ & RC \\
E. benthamii & 0,497 & $0,692^{\mathrm{a}}$ & $1,392^{\mathrm{c}}$ \\
& $(13,26)$ & $(12,56)$ & $(12,56)$ \\
E. dunnii & 0,500 & $0,698^{\mathrm{a}}$ & $1,393^{\mathrm{c}}$ \\
& $(10,93)$ & $(12,67)$ & $(12,67)$ \\
E. grandis & 0,411 & $0,680^{\mathrm{a}}$ & $1,656^{\mathrm{b}}$ \\
& $(8,36)$ & $(10,42)$ & $(10,42)$ \\
E. saligna & 0,493 & $0,695^{\mathrm{a}}$ & $1,410^{\mathrm{c}}$ \\
& $(7,87)$ & $(11,74)$ & $(11,74)$ \\
E. urograndis & 0,472 & $0,695^{\mathrm{a}}$ & $1,472^{\mathrm{c}}$ \\
& $(7,35)$ & $(12,79)$ & $(12,79)$ \\
Mix ${ }^{1}$ & 0,475 & $0,694^{\mathrm{a}}$ & $1,461^{\mathrm{c}}$ \\
& - & $(11,66)$ & $(11,66)$ \\
P. taeda & 0,377 & $0,682^{\mathrm{a}}$ & $1,810^{\mathrm{a}}$ \\
\hline
\end{tabular}

ME = massa específica; ${ }^{1}$ Mistura das 5 espécies de Eucalyptus; $\mathrm{RC}=$ razão de compactação; Médias seguidas de mesma letra na mesma coluna não diferem estatisticamente entre si pelo teste de Tukey ao nível de $5 \%$ de probabilidade; Valores entre parênteses referem-se ao coeficiente de variação em porcentagem.
Para a razão de compactação, o E. grandis apresentou valor médio estatisticamente superior às demais espécies de Eucalyptus, em decorrência da menor massa específica da sua madeira entre as espécies do gênero. As demais espécies de Eucalyptus apresentaram igualdade estatística apresentando razões de compactação semelhantes. Nenhuma das espécies de Eucalyptus apresentou razão de compactação superior ao $P$. taeda, a qual difere estatisticamente das primeiras. Pode-se afirmar que a espécie influenciou os valores de razão de compactação, sendo que a massa específica da madeira é o fator limitante dessa variável; como todas as espécies se diferenciam nesse aspecto, essa diferença é refletida nos valores obtidos para a razão de compactação.

Ainda em relação à razão de compactação, pode-se dizer que, com exceção da testemunha e do E. grandis, todas as espécies apresentaram valor médio de acordo com a faixa descrita como ideal por Maloney (1993), que varia de 1,3 a 1,6 e indica contato satisfatório entre as partículas.

Os valores de $\mathrm{RC}$ obtidos neste estudo foram superiores aos encontrados por autores como: Bufalino et al. (2015), que observaram um valor de 1,29 para E. urograndis, Iwakiri et al. (2004), que obtiveram valores de 1,13,0,87 e 0,97 para, respectivamente, E. grandis, E. dunnii e E. saligna, e Gorski et al. (2015), que observaram uma média de 1,22 para E. benthamii.

\subsection{Propriedades físicas dos painéis}

Os valores médios de absorção d'água e inchamento em espessura após $2 \mathrm{~h}$ e $24 \mathrm{~h}$ de imersão são apresentados na Tabela 3.

Verificou-se que em todas as propriedades avaliadas o comportamento foi semelhante estatisticamente, sendo que os menores valores foram obtidos pelas espécies do gênero Eucalyptus. Dessa forma, é importante salientar que os painéis de Eucalyptus apresentaram menores valores para as propriedades de estabilidade dimensional em comparação aos painéis de Pinus taeda, o que pode ser atribuído à influência exercida da diferença entre gêneros sobre as propriedades de estabilidade dimensional, pois, dentro do gênero Eucalyptus, não houve diferenças estatísticas significativas entre as espécies. Os painéis compostos pelo mix de espécies mostraram-se semelhantes aos demais produzidos com as espécies de Eucalyptus puras, indicando que ambas 
Tabela 3. Valores médios de absorção d'água e inchamento em espessura após 2 h e $24 \mathrm{~h}$.

Table 3. Average of water absorption and thickness swelling after 2 and 24 hours.

\begin{tabular}{|c|c|c|c|c|}
\hline Espécie & $\begin{array}{c}\text { AA } 2 \mathrm{~h} \\
(\%)\end{array}$ & $\begin{array}{c}\text { AA 24h } \\
(\%)\end{array}$ & $\begin{array}{c}\text { IE } 2 \mathrm{~h} \\
(\%)\end{array}$ & $\begin{array}{c}\text { IE 24h } \\
(\%)\end{array}$ \\
\hline E. benthamii & $\begin{array}{l}11,24^{a} \\
(35,55)\end{array}$ & $\begin{array}{l}36,58^{a} \\
(24,82)\end{array}$ & $\begin{array}{c}2,09^{\mathrm{a}} \\
(35,75)\end{array}$ & $\begin{array}{c}9,15^{\mathrm{a}} \\
(26,28)\end{array}$ \\
\hline E. dunnii & $\begin{array}{l}10,86^{\mathrm{a}} \\
(29,45)\end{array}$ & $\begin{array}{l}36,27^{\mathrm{a}} \\
(19,00)\end{array}$ & $\begin{array}{c}2,46^{\mathrm{a}} \\
(30,72)\end{array}$ & $\begin{array}{l}10,44^{\mathrm{a}} \\
(26,67)\end{array}$ \\
\hline E. grandis & $\begin{array}{l}10,37^{a} \\
(28,76)\end{array}$ & $\begin{array}{l}35,29^{a} \\
(22,51)\end{array}$ & $\begin{array}{c}2,44^{\mathrm{a}} \\
(38,00)\end{array}$ & $\begin{array}{c}9,21^{\mathrm{a}} \\
(30,60)\end{array}$ \\
\hline E. saligna & $\begin{array}{l}10,32^{a} \\
(34,93)\end{array}$ & $\begin{array}{l}31,66^{a} \\
(26,46)\end{array}$ & $\begin{array}{c}2,07^{\text {a }} \\
(36,87)\end{array}$ & $\begin{array}{c}7,98^{\mathrm{a}} \\
(29,90)\end{array}$ \\
\hline E. urograndis & $\begin{array}{l}10,49^{a} \\
(38,93)\end{array}$ & $\begin{array}{l}33,29^{a} \\
(28,00)\end{array}$ & $\begin{array}{c}2,20^{\mathrm{a}} \\
(30,66)\end{array}$ & $\begin{array}{c}9,97^{\mathrm{a}} \\
(28,08)\end{array}$ \\
\hline $\operatorname{Mix}^{1}$ & $\begin{array}{l}11,37^{a} \\
(32,84)\end{array}$ & $\begin{array}{l}33,38^{a} \\
(23,43)\end{array}$ & $\begin{array}{c}2,50^{\text {a }} \\
(33,86)\end{array}$ & $\begin{array}{c}8,56^{\mathrm{a}} \\
(24,60)\end{array}$ \\
\hline P. taeda & $\begin{array}{l}22,99^{\mathrm{b}} \\
(29,61)\end{array}$ & $\begin{array}{l}59,55^{\mathrm{b}} \\
(13,31)\end{array}$ & $\begin{array}{c}5,99^{\mathrm{b}} \\
(35,57)\end{array}$ & $\begin{array}{l}21,28^{b} \\
(24,90)\end{array}$ \\
\hline
\end{tabular}

AA $2 \mathrm{~h}$ e $24 \mathrm{~h}=$ absorção dágua após $2 \mathrm{~h}$ e $24 \mathrm{~h}$; IE $2 \mathrm{~h}$ e $24 \mathrm{~h}=$ inchamento em espessura após $2 \mathrm{~h}$ e $24 \mathrm{~h}$; ${ }^{1}$ Mistura das 5 espécies de Eucalyptus; Médias seguidas de mesma letra na mesma coluna não diferem estatisticamente entre si pelo teste de Tukey ao nível de $5 \%$ de probabilidade; Valores entre parênteses referem-se ao coeficiente de variação em porcentagem.

as espécies em mistura podem gerar painéis estáveis quando expostos à umidade.

Os valores obtidos neste estudo para as propriedades de absorção d'água e inchamento em espessura foram inferiores aos encontrados por alguns autores na literatura, como: Iwakiri et al. (2004), que obtiveram valores para AA 2 h de 11,98\%, 38,42\% e 19,31\%, AA 24 h de 28,85\%, 70,96\% e 43,72, IE 2 h de 8,88\%, $34,50 \%$ e $14,20 \%$ e IE 24 h de $15,71 \%, 60,86 \%$ e $28,98 \%$, respectivamente, para as espécies E. grandis, E. dunnii e E. saligna, e Bufalino et al. (2015), que obtiveram valores para AA 2 h de 42,00\%, AA 24 h de 55,00\%, IE 2 h de 13,00\% e IE 24 h de 18,00\% para E. urograndis, e, ainda, Gorski et al. (2015), que obtiveram valores para AA 2 h de 19,30\%, AA 24 h de 60,50\%, IE 2 h de 9,00\% e IE 24 h de 32,01\% para E. benthamii. Contudo, nesses estudos os autores também observaram que os painéis produzidos com Eucalyptus se apresentaram mais estáveis do que aqueles produzidos com Pinus.

Em comparação com os valores máximos sugeridos pela norma EN 300 (CEN, 2006) para inchamento após 24 horas de imersão de painéis com espessura entre $10 \mathrm{~mm}$ e $18 \mathrm{~mm}$, observa-se que todos os tratamentos (puros e em mistura) produzidos com as espécies de
Eucalyptus atendem os requisitos para painéis OSB 1 (painéis não estruturais de uso geral e interior); OSB 2 (painéis estruturais para uso em condições secas), OSB 3 (painéis estruturais para uso em condições úmidas) e OSB 4 (painéis estruturais de carregamento pesado para uso em condições úmidas), que exigem inchamento máximo de $25 \%$, 20\%, $15 \%$ e $12 \%$, respectivamente, permitindo dessa forma ampla gama de aplicações. Já o tratamento testemunha ( $P$. taeda) atende somente o requisito de painéis OSB 1, o qual, nessas condições experimentais, restringe-se às aplicações não estruturais de uso geral e interior.

\subsection{Propriedades mecânicas dos painéis}

Os valores médios dos módulos de ruptura e elasticidade nos sentidos paralelo e perpendicular, e da tração perpendicular à superfície dos painéis, são apresentados na Tabela 4 .

Pode-se afirmar que a propriedade de MOR tanto no sentido paralelo como no perpendicular não foi influenciada pela espécie. Por outro lado, o MOE em ambos os sentidos sofreu uma influência significativa do gênero e espécie, onde Pinus taeda mostrou-se estatisticamente inferior às espécies de Eucalyptus.

Autores como Iwakiri et al. (2004), assim como neste estudo, não encontraram diferenças significativas para MOR entre algumas espécies de Eucalyptus e o Pinus taeda. Os mesmos autores atribuem esse comportamento à alta razão de compactação dos painéis obtidos em virtude da baixa densidade da madeira, como no caso do Pinus taeda e do E. grandis. Os autores ressaltam, ainda, que o fato de as partículas strand apresentarem maiores dimensões e geometria pode favorecer o contato superficial e a ligação entre as partículas, gerando painéis com propriedades mecânicas satisfatórias mesmo naqueles com menor razão de compactação.

O mix de espécies demonstrou que a interação entre as espécies proporcionou resistência semelhante aos painéis produzidos com as espécies puras; assim sendo, é viável que se produzam painéis compostos com a mistura dessas espécies em mesmas proporções, para que se obtenham painéis de boa resistência e apresentando a vantagem de maior variabilidade e disponibilidade de matéria-prima.

Observou-se que a espécie testemunha ( $P$. taeda) apresentou o maior valor médio para resistência à 
Tabela 4. Valores médios das propriedades mecânicas dos painéis.

Table 4. Average values of mechanical properties of panels.

\begin{tabular}{|c|c|c|c|c|c|}
\hline \multirow[b]{2}{*}{ Espécie } & \multicolumn{2}{|c|}{ Paralelo } & \multicolumn{2}{|c|}{ Perpendicular } & \multirow{2}{*}{$\begin{array}{c}\text { Tração } \\
\text { perpendicular } \\
(\mathrm{MPa})\end{array}$} \\
\hline & $\begin{array}{l}\text { MOR } \\
(\mathrm{MPa})\end{array}$ & $\begin{array}{l}\text { MOE } \\
\text { (MPa) }\end{array}$ & $\begin{array}{l}\text { MOR } \\
(\mathrm{MPa})\end{array}$ & $\begin{array}{l}\text { MOE } \\
\text { (MPa) }\end{array}$ & \\
\hline E. benthamii & $\begin{array}{l}25,63^{a} \\
(33,79)\end{array}$ & $\begin{array}{c}4902,83^{a} \\
(20,46)\end{array}$ & $\begin{array}{l}27,47^{a} \\
(26,71)\end{array}$ & $\begin{array}{c}2884,19^{a} \\
(19,43)\end{array}$ & $\begin{array}{c}0,56^{\mathrm{bc}} \\
(28,89)\end{array}$ \\
\hline E. dunnii & $\begin{array}{l}23,90^{a} \\
(48,58)\end{array}$ & $\begin{array}{c}5184,67^{a} \\
(27,85)\end{array}$ & $\begin{array}{l}26,11^{\mathrm{a}} \\
(31,48)\end{array}$ & $\begin{array}{c}2855,50^{a} \\
(17,34)\end{array}$ & $\begin{array}{c}0,47^{c} \\
(25,80)\end{array}$ \\
\hline E. grandis & $\begin{array}{l}26,80^{\mathrm{a}} \\
(30,99)\end{array}$ & $\begin{array}{c}4920,96^{\mathrm{a}} \\
(20,91)\end{array}$ & $\begin{array}{l}24,45^{\mathrm{a}} \\
(21,15)\end{array}$ & $\begin{array}{c}2823,78^{\mathrm{a}} \\
(18,93)\end{array}$ & $\begin{array}{l}0,52^{\mathrm{bc}} \\
(24,87)\end{array}$ \\
\hline E. saligna & $\begin{array}{l}29,92^{a} \\
(29,00)\end{array}$ & $\begin{array}{c}5588,01^{a} \\
(21,65)\end{array}$ & $\begin{array}{l}30,60^{\mathrm{a}} \\
(22,55)\end{array}$ & $\begin{array}{c}3220,68^{a} \\
(18,27)\end{array}$ & $\begin{array}{c}0,54^{b c} \\
(23,13)\end{array}$ \\
\hline E. urograndis & $\begin{array}{l}27,67^{a} \\
(40,20)\end{array}$ & $\begin{array}{c}5520,30^{\mathrm{a}} \\
(24,31)\end{array}$ & $\begin{array}{l}25,11^{\mathrm{a}} \\
(28,50)\end{array}$ & $\begin{array}{c}2976,09^{a} \\
(16,76)\end{array}$ & $\begin{array}{c}0,45^{c} \\
(28,06)\end{array}$ \\
\hline $\operatorname{Mix}^{1}$ & $\begin{array}{l}30,70^{a} \\
(29,77)\end{array}$ & $\begin{array}{c}5474,31^{a} \\
(20,98)\end{array}$ & $\begin{array}{l}29,30^{\mathrm{a}} \\
(32,96)\end{array}$ & $\begin{array}{c}3195,96^{\mathrm{a}} \\
(33,08)\end{array}$ & $\begin{array}{c}0,59^{\mathrm{b}} \\
(21,78)\end{array}$ \\
\hline P. taeda & $\begin{array}{l}29,97^{a} \\
(32,57)\end{array}$ & $\begin{array}{c}3995,03^{b} \\
(23,71)\end{array}$ & $\begin{array}{l}24,73^{\mathrm{a}} \\
(29,15)\end{array}$ & $\begin{array}{c}2247,25^{b} \\
(20,07)\end{array}$ & $\begin{array}{c}0,74^{\mathrm{a}} \\
(24,56)\end{array}$ \\
\hline
\end{tabular}

$\mathrm{MOR}=$ módulo de ruptura; $\mathrm{MOE}=$ módulo de elasticidade; ${ }^{1}$ Mistura das 5 espécies de Eucalyptus; Médias seguidas de mesma letra na mesma coluna não diferem estatisticamente entre si pelo teste de Tukey ao nível de $5 \%$ de probabilidade; Valores entre parênteses referem-se ao coeficiente de variação em porcentagem.

tração, diferindo estatisticamente das espécies de Eucalyptus. Apesar de diferenças entre algumas espécies de Eucalyptus, estas obtiveram valores muito próximos de resistência à tração entre si. Por outro lado, as espécies E. dunnii e E. urograndis apresentaram os menores valores para a propriedade.

Na literatura encontram-se autores como Gouveia (2001), Iwakiri et al. (2004) e Gorski (2014) que também obtiveram valores de tração para Pinus superiores aos de Eucalyptus. Painéis com alta razão de compactação, como é o caso daqueles produzidos com P. taeda, apresentam melhor contato entre partículas devido à maior compressão obtida durante a prensagem, o que pode ter oferecido aos painéis melhores resultados de resistência à tração. Por outro lado, Gorski (2014) vincula esse fato também à maior porosidade de madeira dessa espécie em relação às de Eucalyptus, favorecendo o espalhamento e a penetração da resina, oferecendo melhor colagem interna dos painéis.

O fato de as espécies E. dunnii e E. urograndis apresentarem os menores valores para a propriedade em questão pode estar relacionado a alguma característica química e anatômica das espécies, porém não se pode afirmar, sendo que neste estudo não se objetivou a caracterização química e anatômica das espécies.

Afirma-se desse modo que a espécie exerce influência sobre a resistência à tração dos painéis, porém essa questão está mais ligada às diferenças de massa específica da madeira obtidas para cada uma, o que leva a diferentes resultados de razão de compactação e determina o grau de contato entre as partículas na prensagem do colchão.

Vale ressaltar que o mix de espécies obteve desempenho estatisticamente semelhante às espécies E. benthamii, E. grandis, E. saligna para essa propriedade, assim como em outras anteriormente discutidas, desse modo a mistura das espécies proporcionou aos painéis resultados satisfatórios de tração perpendicular. Assim sendo, afirma-se que a mistura das cinco espécies nas condições deste estudo pode ser utilizada de forma efetiva para a produção de painéis OSB, a fim de obter um produto com boa qualidade assim como aqueles produzidos com espécies puras, porém utilizando-se de matéria-prima diversificada.

Considerando a norma EN 300 (CEN, 2006), verificou-se que os tratamentos produzidos com o E. saligna e o mix atingiram a resistência e a rigidez mínimas de $28 \mathrm{MPa}$ e $4.800 \mathrm{MPa}$ para o sentido paralelo, e de $15 \mathrm{MPa}$ e $1.900 \mathrm{MPa}$, para o sentido perpendicular, além de resistência à tração perpendicular de $0,45 \mathrm{MPa}$, sendo dessa forma classificados como OSB tipo 4, que permite aplicações estruturais de carregamento pesado para uso em condições úmidas. Já os demais tratamentos (E. benthamii, E. dunnii, E. grandis, E. urograndis e 
P. taeda) são classificados como OSB tipo 3, cujos requisitos mínimos são de: $\mathrm{MOR}$ paralelo: $20 \mathrm{MPa}$, MOE paralelo: $3.500 \mathrm{MPa}$, MOR perpendicular: $10 \mathrm{MPa}$ e MOE perpendicular: $1.400 \mathrm{MPa}$, e tração perpendicular de 0,32 $\mathrm{MPa}$, podendo ser utilizados estruturalmente em condições secas e úmidas.

Vale ressaltar ainda que todos os tratamentos podem ser utilizados em aplicações do tipo OSB 1, ou seja, como painéis não estruturais de uso geral e interior seco (MOR paralelo: $18 \mathrm{MPa}$, MOE paralelo: $2.500 \mathrm{MPa}$, MOR perpendicular: $9 \mathrm{MPa}$, MOE perpendicular: $1.200 \mathrm{MPa}$, e tração perpendicular: 0,28 MPa), e em painéis OSB tipo 2, que permitem utilização como painéis estruturais para uso em condições secas (MOR paralelo: 20 MPa, MOE paralelo: $3.500 \mathrm{MPa}$, MOR perpendicular: $10 \mathrm{MPa}$, MOE perpendicular: 1.400 MPa, e tração perpendicular: 0,32 MPa).

\section{CONCLUSÕES}

Com base nos resultados obtidos neste estudo pode-se concluir que:

As espécies de Eucalyptus avaliadas, provenientes de ciclo de curta rotação, mostraram-se viáveis para a produção de painéis OSB.

A mistura das espécies de Eucalyptus apresentou resultados satisfatórios, oferecendo boas propriedades aos painéis OSB, tornando viável a sua utilização nas condições deste estudo.

Os painéis produzidos com as espécies do gênero Eucalyptus apresentaram, com exceção do MOR e tração perpendicular, propriedades superiores em relação aos painéis produzidos com a testemunha $P$. taeda, espécie tradicionalmente utilizada pela indústria brasileira.

Os tratamentos produzidos com a espécie E. saligna e a partir da mistura de espécies foram classificados como OSB tipo 4, que permite aplicações estruturais de carregamento pesado para uso em condições úmidas, e os demais tratamentos como OSB tipo 3, cujas aplicações são estruturais em condições secas e úmidas.

Além das classificações mencionadas anteriormente, todos os tratamentos podem ser utilizados para finalidades não estruturais de uso geral e interior seco (OSB 1), e como painéis estruturais para uso em condições secas (OSB 2).

\section{STATUS DA SUBMISSÃO}

Recebido: 29 abr., 2016

Aceito: 1 out., 2016

\section{AUTOR(ES) PARA CORRESPONDÊNCIA}

\section{Thiago Souza da Rosa}

Universidade Federal do Paraná - UFPR

Av. Pref. Lothário Meissner, 632, CEP 80060-000, Curitiba, PR, Brazil

e-mail: thiagosouzadarosa@gmail.com

\section{REFERÊNCIAS}

Associação Brasileira de Produtores de Florestas Plantadas - ABRAF. Anuário estatístico 2013, Ano base 2012, Brasília, 2013. 142 p.

Bufalino L, Corrêa AAR, Sá VA, Mendes LM, Almeida NA, Pizzol VD. Alternative compositions of Oriented Strand Boards (OSB) made with commercial woods produced in Brazil. Maderas: Ciencia y Tecnología 2015, 17(1): 105-116.

Cloutier A. Oriented strandboard (OSB): raw material, manufacturing process, properties of wood-base fiber and particle materials. In: Proceedings of the International Seminar on Solid Wood Products of High Technology; 1998; Belo Horizonte. Belo Horizonte: SIF; 1998. p. 173-185.

European Committee for Standardization - CEN. EN 310. Determination of modulus of elasticity in bending and of bending strength. Bruxelas: CEN; 2002a.

European Committee for Standardization - CEN. EN 317. Determination of swelling in thickness after immersion in water. Bruxelas: CEN; 2002 b.

European Committee for Standardization - CEN. EN 319. Perpendicular tensile strength of particleboards and fiberboards. Bruxelas: CEN; 2002c.

European Committee for Standardization - CEN. EN 323. Wood based panels - Determination of density. Bruxelas: 2002d.

European Committee for Standardization - CEN. EN 300: Oriented Strand Boards (OSB) - Definitions, classification and specifications. Bruxelas: CEN; 2006.

Gorski L, Cunha AB, Rios PD, Trianoski R, Martarello LP. Produção de painéis de partículas orientadas (OSB) de Eucalyptus benthamii e Pinus spp. em diferentes combinações de camadas. Scientia Forestalis 2015; 43(106): 367-375.

Gorski L. Painéis de partículas orientadas (OSB) da madeira de Pinus spp. e Eucalyptus benthamii [dissertação]. Lages: Universidade do Estado de Santa Catarina; 2014. 
Gouveia FN. Produção de chapas de partículas orientadas (OSB) a partir das espécies Eucalyptus grandis W. Hill ex Maiden e Pinus elliottii Engelm [dissertação]. Viçosa: Universidade Federal de Viçosa; 2001.

Irle M, Barbu MC. Wood-based panel technology. In: Thoemen H, Irle M, Sernek M. Wood-based panels: an introduction for specialists. London: Brunel University Press; 2010. p. 1-90.

Iwakiri S, Albuquerque CEC, Prata JG, Costa ACB. Utilização de madeiras de Eucalyptus grandis e Eucalyptus dunnii para produção de painéis de partículas orientadas - OSB. Ciência Florestal 2008; 18(2): 265-270. http:// dx.doi.org/10.5902/19805098463.

Iwakiri S, Mendes LM, Saldanha LK, Santos JC. Utilização da madeira de eucalipto na produção de chapas de partículas orientadas OSB. Cerne 2004; 10(1): 46-52.

Iwakiri S, Silva JRM, Matoski SLS, Leonhadt G, Caron J. Produção de chapas de madeira aglomerada de cinco espécies de pinus tropicais. Floram 2001; 8(1): 137-142.

Maloney TM. Modern particleboard e dry-process fiberboard manufacturing. 2nd ed. São Francisco: Miller Freeman Publication; 1993.
Melo JE, Coradin VTR, Mendes JC. Classes de densidade para madeiras da Amazônia Brasileira. In: Anais do $6^{\circ}$ Congresso florestal Brasileiro, Florestas e Meio ambiente: Conservação e Produção, Patrimônio Social; 1990; Campos do Jordão. Campos do Jordão: Sociedade Brasileira de Silvicultura; Sociedade Brasileira de Engenheiros Florestais; 1990. p. 695-699.

Mendes RF, Mendes LM, Mendonça LM, Guimarães JB Jr, Mori FA. Qualidade de painéis aglomerados homogêneos produzidos com a madeira de clones de Eucalyptus urophylla. Cerne 2014; 20(2): 329-336. http://dx.doi.org /10.1590/01047760.201420021273.

Mendes RF. Efeito do tratamento térmico sobre as propriedades de painéis OSB [dissertação]. Piracicaba: Escola Superior de Agricultura Luiz de Queiroz; 2010.

Sistema Nacional de Informações Florestais - SNIF. As florestas plantadas[online]. Brasília: SNIF; 2013. [citado em 2015 mar. 6]. Disponível em: http://www.florestal.gov. $\mathrm{br} /$ snif/recursos-florestais/as-florestas-plantadas

Vidal ACF, Hora AB. Panorama de mercado: painéis de madeira. BNDES Setorial 2014; 40: 323-384. 